\title{
Shape of transition layers in a differential-delay equation
}

\author{
Jonathan AD Wattis \\ School of Mathematical Sciences, University of Nottingham, \\ University Park, Nottingham, NG7 2RD, UK
}

April 19, 2017

\begin{abstract}
We use asymptotic techniques to describe the bifurcation from steady-state to a periodic solution in the singularly perturbed delayed logistic equation $\varepsilon \dot{x}(t)=-x(t)+\lambda f(x(t-1))$ with $\varepsilon \ll 1$. The solution has the form of plateaus of approximately unit width separated by narrow transition layers. The calculation of the period two solution is complicated by the presence of delay terms in the equation for the transition layers, which induces a phase shift that has to be calculated as part of the solution. High order asymptotic calculations enable both the shift and the shape of the layers to be determined analytically, and hence the period of the solution. We show numerically that the form of transition layers in the four-cycles is similar to that of the two-cycle, but that a three-cycle exhibits different behaviour. Asymptotic analysis, Differential-delay equation, transition layers
\end{abstract}

\section{Introduction}

The differential-delay equation (DDE)

$$
\varepsilon \frac{\mathrm{d} x}{\mathrm{~d} t}=-x(t)+f(x(t-1)),
$$

has been used by a variety of authors to model a wide range of physical phenomena, from population dynamics, as discussed by Gurney et al (1980), to physiology, where the production of red blood cells has been described by Mackey (1979); Mackey \& Glass (1977); Glass \& Mackey (1988) and WazewskaCzysewska \& Lasota (1976), to the behaviour of an optical cavity resonator, as studied by Ikeda (1979, 1985); Ikeda et al. (1980). In all these cases, the feedback function $f(\cdot)$ is nonlinear, having the form of a 'humped' function, that is, as $x$ increases, $f$ rises to a maximum and then decays. Variants of this system include a delayed logistic equation of the form $\mathrm{d} x / \mathrm{d} t=\lambda x(t)[1-x(t-1)]$, analysed by Fowler (1982), who used asymptotic methods to explain the periodic solution of large amplitude spikes separated by long small amplitude plateaus.

Equation (1.1) has been analysed using a range of mathematical techniques, for example, Erneux et al. (2004) analyse the bifurcation to periodic solutions in the Ikeda system. Chow \& Mallet-Paret (1983) investigate chaotic behaviour in singularly perturbed differential-delay equations. Hale \& Huang (1994) proved the existence of periodic orbits of (1.1) in certain parts of parameter space. The form of periodic solutions has been investigated by Chow et al. (1992) who note that as $\varepsilon \rightarrow 0$, the waves become square, and have period approximately equal to two. Fowler \& Mackey (2002) also note that the limit $\varepsilon \ll 1$ is singular, and use asymptotic analysis to describe the periodic solution in terms of relaxation oscillations.

Various properties about the form of the solution in the large time limit, and properties of the convergence to the square wave in the limit $\varepsilon \rightarrow 0$ have been rigorously established by Mallet-Paret and 
Nussbaum. Nussbaum (1982) proved the existence of slowly-oscillating periodic solutions. Bounds on their shape were established by Mallet-Paret \& Nussbaum (1986), together with the fact that there may be multiple periodic solutions and multiple extrema in each period of oscillation. Mallet-Paret \& Nussbaum (1986) went on to prove that the period of the two cycle is $2+\mathscr{O}(\varepsilon)$ and, by considering a coupled problem for the two transition layer functions of the two-cycle, they establish bounds on the overshooting behaviour. They note that such behaviour is reminiscent of Gibbs phenomena in truncated Fourier series. The dependence of qualitative properties of the solution $x(t)$ on the form of the nonlinearity $f(\cdot)$ was analysed by Mallet-Paret \& Nussbaum (1989); in this paper they discuss how the solution profile approximates the square wave solution in the limit $\varepsilon \rightarrow 0$ for several example functions $f(\cdot)$. Mallet-Paret \& Nussbaum (1993) consider the DDE (1.1) in the case where $f(\cdot)$ is a step function, and show that in this case solutions $x(t)$ do not converge to solutions of $x_{n+1}=f\left(x_{n}\right)$ in the limit $\varepsilon \rightarrow 0$, but rather to a modified map. In contrast to this rigorous work, the approach taken here is to use asymptotic techniques to give simple explicit expressions for the shape of the transition layers.

Adhikari et al. (2008) also perform an asymptotic expansion of the waveform, obtaining an approximation in terms of elliptic functions and an expression for the period. Using global continuation of heteroclinic orbits, Chow et al. (1989) prove that transition layers in the periodic orbit are monotone, provided the nonlinearity $f(x)$ is monotone. Fowler (1997) speculates that the onset of chaos is associated with a homoclinic connection of a periodic orbit in phase space.

These examples and analyses use a variety of nonlinear feedback functions, $f(x)$; in this paper we focus on the simplest case, where $f(x)=\lambda x(1-x)$, which corresponds to the logistic map. This function is chosen for its mathematical simplicity, as it allows the period two cycle of the underlying difference equation to be explicitly determined.

In the limit $\varepsilon \rightarrow 0$, the differential delay equation (1.1) reduces to the logistic map, whose properties we summarise in the remainder of this section. We also review the Hopf bifurcations in the DDE which occur for fixed $\lambda$ as $\varepsilon>0$ is reduced, and which lead to smooth periodic solutions. In section 2 , we show that when $\varepsilon=0$ the solution $x(t)$ has the form of a sequence of plateaus separated by discrete jumps. For $0<\varepsilon \ll 1$, these plateaus are connected by narrow transition layers. Thus the bifurcation has quite distinct properties which the standard Hopf analysis fails to explain. It is the purpose of this paper to describe this bifurcation in more detail and, in particular, to analyse the form of these transition layers. In order to facilitate analysis of the transition layers, we introduce a reformulation of the problem, both for the general transition layer, and in particular for the two-cycle. In section 3 we construct asymptotic approximations to the solutions obtained when $\varepsilon \ll 1$ and $\lambda$ is increased through $\lambda=3$, which is the bifurcation point where the period two solution is created. Our analysis of this case shares some similarity with that of Adhikari et al. (2008); however, we avoid the use of elliptic functions. Section 4 contains a numerical investigation of the transition layers in the period 3 and period 4 cycles. In Section 5 we conclude the paper with a discussion of the key results.

\subsection{Properties of the map}

When $\varepsilon=0$ the differential-delay equation (1.1) reduces to the logistic map

$$
x_{n+1}=\lambda x_{n}\left(1-x_{n}\right),
$$

which has been extensively studied, for example by May (1976) and Holton \& May (1993). Here we summarise the results and properties of this map that are relevant to our later calculations, quoting the behaviour which is observed in each range of $\lambda$ values:

- for $0 \leq \lambda \leq 1$, there is only one fixed point in $[0,1]$, namely $x=0$, and this is stable.

- for $1<\lambda \leq 3$, there are two fixed points, namely $x=0$, which is unstable, and $x=1-1 / \lambda$, which is stable. 
- for $3<\lambda$, the two fixed points $x=0$ and $x=1-1 / \lambda$ are both unstable. In this parameter regime, there is a two-cycle, given by

$$
x_{ \pm}=\frac{1}{2 \lambda}[\lambda+1 \pm \sqrt{(\lambda-3)(\lambda+1)}]
$$

- for $3<\lambda \leq 1+\sqrt{6} \approx 3.449$ the two-cycle (1.3) is stable and is thus the attractor for the large $n$ iterates. In this paper, we are predominantly interested in this range of $\lambda$.

- for $1+\sqrt{6}<\lambda<3.544 \ldots$ the two fixed points and the two-cycle (1.3) are all unstable, and a fourcycle forms the stable long-time attractor.

- as $\lambda$ increases further $(\lambda>3.544)$, there is a succession of bifurcations to increasingly complex behaviour.

\subsection{Hopf bifurcation}

Chow \& Mallet-Paret (1983) consider the differential-delay equation (1.1) and show that there is a Hopf bifurcation curve in $(\lambda, \varepsilon)$-parameter space. We reproduce these results here, partly for the sake of completeness, but mainly to illustrate the qualitative difference between (i) the bifurcation and the form of solution obtained when one fixes $\lambda>3$ and reduces $\varepsilon$, as Chow and Mallet-Paret consider, and, (ii) the case when $0<\varepsilon \ll 1$ and $\lambda$ increases through the value $\lambda=3$, which we focus on in Section 3 .

Here we make no specific assumptions on the values of $\lambda, \varepsilon$, we treat them both as $\mathscr{O}(1)$ parameters. We seek the region of parameter space where the uniform solution $x_{s}=1-1 / \lambda$ is stable, by substituting $x=1-1 / \lambda+\delta \mathrm{e}^{\gamma t+i \omega t}$ into the governing equation (1.1) with $\gamma, \omega \in \mathbb{R}$ and taking $\delta \ll 1$. At $\mathscr{O}(\delta)$ we obtain

$$
\varepsilon \gamma+1=-(\lambda-2) \mathrm{e}^{-\gamma} \cos \omega, \quad \varepsilon \omega=(\lambda-2) \mathrm{e}^{-\gamma} \sin \omega
$$

This system of equations for $\gamma, \omega$ can be rearranged to give

$$
\tan \omega=\frac{-\varepsilon \omega}{1+\varepsilon \gamma}, \quad(1+\varepsilon \gamma)^{2}+\varepsilon^{2} \omega^{2}=(\lambda-2)^{2} \mathrm{e}^{-2 \gamma}
$$

A bifurcation occurs when the growth rate, $\gamma$, changes sign: the curve on which $\gamma=0$ is given parametrically by

$$
\varepsilon=-\frac{1}{\omega} \tan \omega, \quad \lambda=2-\sec \omega .
$$

For small $\varepsilon$, solutions of the former equation can be approximated by $\omega=n \pi\left(1-\varepsilon+\varepsilon^{2}\right)$ for any $n \in \mathbb{N}$. Substituting this approximation into the latter expression of (1.6) leads to a family of curves in $(\lambda, \varepsilon)$ parameter space which are approximated by

$$
\lambda_{n}(\varepsilon)=3+\frac{1}{2} n^{2} \pi^{2} \varepsilon^{2}, \quad \text { or } \quad \varepsilon_{n}(\lambda)=\frac{1}{n \pi} \sqrt{2(\lambda-3)}, \quad \text { for odd } n .
$$

The first few curves are illustrated in Figure 1. The solid curve corresponds to the primary Hopf bifurcation, $n=1$; the curves corresponding to $n=3,5, \ldots$ yield solutions with higher frequencies.

As well as finding the location of the Hopf bifurcation in $(\lambda, \varepsilon)$ parameter space, this method enables us to explicitly find an approximation for the solution, $x(t)$. Fixing $\lambda$ with $0<\lambda-3 \ll 1$, and reducing $\varepsilon$ so that only the primary $(n=1)$ Hopf bifurcation curve is crossed, in (1.7), that is,

$$
\frac{1}{3 \pi} \sqrt{2(\lambda-3)}=\varepsilon_{3}(\lambda)<\varepsilon<\varepsilon_{1}(\lambda)=\frac{1}{\pi} \sqrt{2(\lambda-3)},
$$




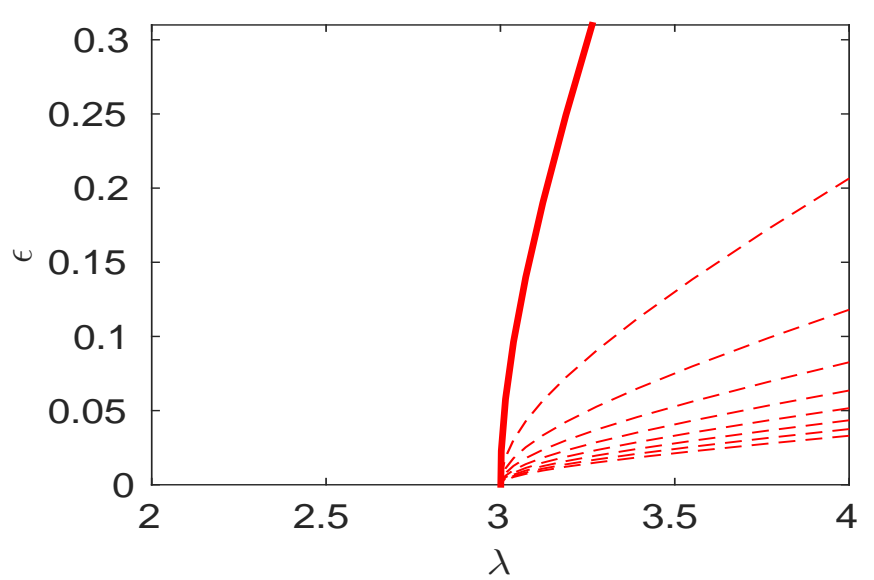

Figure 1: Illustration of the first few Hopf curves in $(\lambda, \varepsilon)$ parameter space, as given by (1.6). To the left of the solid line, the steady-state is stable. The solid line represents the primary Hopf bifurcation curve. The dashed curves represent higher frequency instabilities which mean that when $\varepsilon$ is arbitrarily small, the transition that occurs as $\lambda$ increases from $\lambda<3$ to $\lambda>3$ involves crossing many Hopf curves almost simultaneously.

results in a solution of the form

$$
x(t) \sim 1-\lambda^{-1}+\alpha \sin (\pi t(1-\varepsilon)),
$$

with $\alpha \ll 1$. On the bifurcation curve, the period of the oscillation, $P$, is given by the frequency $\omega$, which corresponds to $n=1$, namely

$$
P=\frac{2 \pi}{\omega} \sim 2+2 \varepsilon=2+\frac{2}{\pi} \sqrt{2(\lambda-3)},
$$

with $\varepsilon \ll 1$. If $\varepsilon$ is reduced further, so that several $(1 \leq n \leq J)$ other Hopf curves are crossed, that is, $\varepsilon$ is given by

$$
\frac{1}{(2 J+1) \pi} \sqrt{2(\lambda-3)}=\varepsilon_{2 J+1}<\varepsilon<\varepsilon_{2 J-1}=\frac{1}{(2 J-1) \pi} \sqrt{2(\lambda-3)},
$$

then a solution of the form

$$
x(t)=1-\frac{1}{\lambda}+\sum_{j=1}^{J} \alpha_{j} \sin ((2 j-1) \pi t(1-\varepsilon)),
$$

is obtained, which has the form of a truncated Fourier series. These series are known to be subject to Gibbs phenomenon, which is a term that describes the overshooting behaviour occurring when a discontinuous function is approximated by a Fourier series, for more details, see Gibbs (1898, 1899); Arfken (1985).

It should be noted that the solution (1.12) has been generated by fixing $\lambda>3$ and reducing $\varepsilon$, crossing several of the Hopf curves illustrated in Figure 1. In the next section, we analyse the less standard case of fixed $\varepsilon>0$ with $\varepsilon \ll 1$ and increasing $\lambda$ through the value $\lambda=3$. Since a large number of Hopf curves are crossed almost simultaneously, the description of the resulting periodic orbit is complicated by the nonlinear terms. In Section 3, we propose a different method of describing the transition from a stable steady-state solution to an oscillatory state which occurs in this case. 

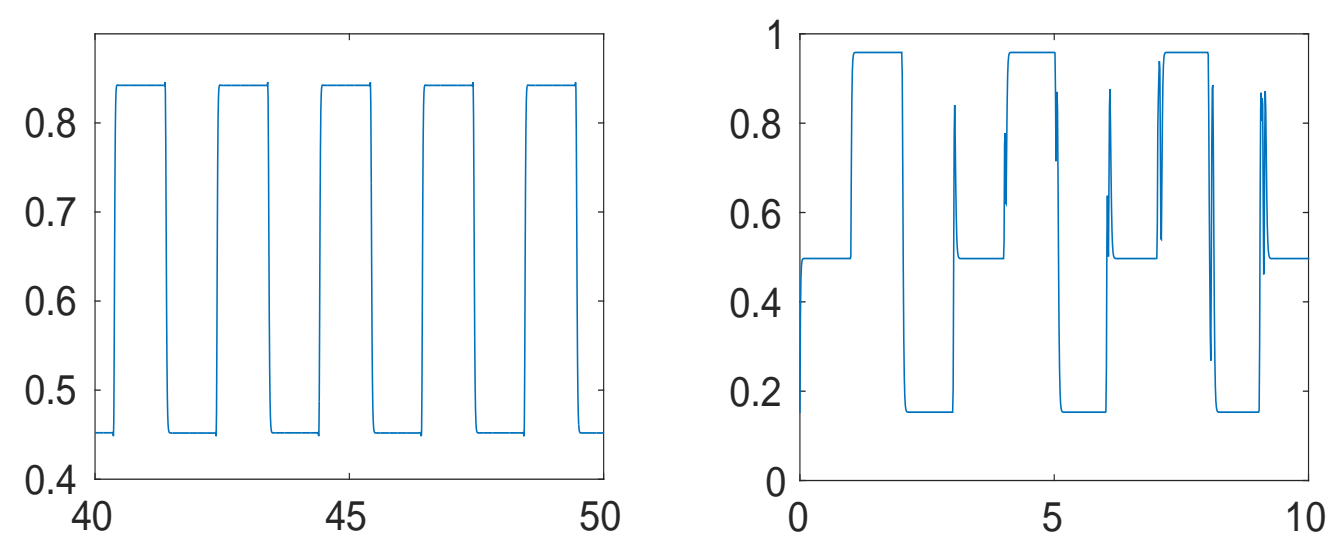

Figure 2: Illustration of a numerical solution of the $\operatorname{DDE}$ (1.1), for the case $\lambda=3.4$ (left) and $\lambda=3.8335$ (right); in both cases, $\varepsilon=0.01$.

\section{Preliminaries}

\subsection{General numerical results}

Figure 2 shows a numerical solution, $x(t)$, of (1.1) for two values of $\lambda$ (produced using matlab solver dde23). The left panel shows a periodic solution with alternating high and low plateaus - a period 2 solution, which occurs when $\lambda=3.4$ and $\varepsilon=0.01$. There are sharp transition layers between the two plateaus, $x_{-}$and $x_{+}$with $x_{ \pm}$given by (1.3), so that $x_{+}=f\left(x_{-}\right)$and $x_{-}=f\left(x_{+}\right)$. When $\varepsilon=0$, that is, for the one-dimensional map (1.2), this solution is stable for $3<\lambda<1+\sqrt{6}$. When the solution of the DDE (1.1) is considered with $\varepsilon>0$, the period of the oscillation is greater than two.

The right-hand panel of Figure 2, illustrates the solution of (1.1) when $\lambda=3.8335$, a value which corresponds to the stable 3-cycle of the one-dimensional map (1.2). In the DDE (1.1), the plateaus clearly follow the 3-cycle; however, in the DDE (1.1) the intervening transition layers do not show any form of periodicity, instead they become increasingly complicated, gaining both in their width and the number of oscillations. Thus the transition layers clearly show an instability. Our aim is to describe the initial stage of this development of complexity in the transition layers.

\subsection{An approximate Poincaré map}

In order to describe and analyse the form of the transition layer, we rescale the time variable so that changes within the layers occur on an $\mathscr{O}(1)$ timescale. To achieve this, we write $t=n+\varepsilon \tau$, so that $\varepsilon \mathrm{d} / \mathrm{d} t=\mathrm{d} / \mathrm{d} \tau$, and we describe each layer via a different function $\psi_{n}(\tau)=x(t)$, with $n=0,1,2, \ldots$. Thus the DDE (1.1) can be rewritten as

$$
\psi_{n}(\tau)+\frac{\mathrm{d} \psi_{n}(\tau)}{\mathrm{d} \tau}=\lambda \psi_{n-1}(\tau)\left(1-\psi_{n-1}(\tau)\right)
$$

We note that this transformation completely removes $\varepsilon$ from the problem.

The form of $\psi_{n}(\tau)$ is such that for $\tau=\mathscr{O}(1), \psi_{n}(\tau)$ describes the $n^{\text {th }}$ transition layer. For large positive and negative $\tau$, with $1 \ll|\tau| \ll \varepsilon^{-1}, \psi_{n}(\tau)$ will be a constant, with potentially different constants at large positive and large negative values of $\tau$. For $1 \ll \tau \ll 1 / \varepsilon$, we have

$$
\psi_{n}(-\tau) \sim \psi_{-\infty}, \quad \psi_{n}(\tau) \sim \psi_{+\infty}, \quad \text { with } \quad \psi_{+\infty}=\lambda \psi_{-\infty}\left(1-\psi_{-\infty}\right) .
$$

We make a distinction between $\tau \rightarrow \infty$ and $1 \ll \tau \ll 1 / \varepsilon$ since $\tau=\mathscr{O}(1 / \varepsilon)$ corresponds to subsequent (or previous) layers, before which, $\psi_{n}$ has relaxed to the constants given in (2.2) with exponentially small 
corrections. At leading order, the matching conditions can then be written in the form

$$
\begin{aligned}
& \psi_{n}(\tau) \rightarrow \psi_{-\infty}^{(n)}, \quad \text { as } \quad \tau \rightarrow-\infty, \quad \text { and } \\
& \psi_{n}(\tau) \rightarrow \psi_{+\infty}^{(n)}=\lambda \psi_{-\infty}^{(n)}\left(1-\psi_{-\infty}^{(n)}\right) \text { as } \tau \rightarrow+\infty .
\end{aligned}
$$

In addition, we have $\psi_{+\infty}^{(n-1)}=\psi_{-\infty}^{(n)}$ and $\psi_{-\infty}^{(n+1)}=\psi_{+\infty}^{(n)}$.

The solution of (2.1) is

$$
\psi_{n}(\tau)=\psi_{n}(\sigma) \mathrm{e}^{\sigma-\tau}+\lambda \mathrm{e}^{-\tau} \int_{\sigma}^{\tau} \mathrm{e}^{s} \psi_{n-1}(s)\left[1-\psi_{n-1}(s)\right] \mathrm{d} s,
$$

for arbitrary $\sigma$, which, in the limit $\sigma \rightarrow-\infty$, leads to

$$
\psi_{n}(\tau)=\lambda \mathrm{e}^{-\tau} \int_{-\infty}^{\tau} \mathrm{e}^{s} \psi_{n-1}(s)\left[1-\psi_{n-1}(s)\right] \mathrm{d} s:=\mathscr{F}\left[\psi_{n-1}(\tau)\right] .
$$

Due to its integral form, this version of the Poincare map may be useful for numerical simulations; however, our asymptotic analysis presented later will use the formulation (2.1). We will call the map $\psi_{n}=\mathscr{F}\left[\psi_{n-1}\right]$ defined by (2.1) or (2.5) the 'fast map' since it describes the shape of the transition layers which occur on the fast timescale. This has a similar form to a map used by Mallet-Paret \& Nussbaum (1993).

\subsection{Simulations of the 2-cycle's transition layers}

For $0<\varepsilon \ll 1$ and $3<\lambda<1+\sqrt{6}$, we observe from numerical simulations of $x(t)$, that there is a periodic solution, with period slightly larger than two. In figure 3 we illustrate the transition layers $\psi_{n}(\tau)$ as calculated using (2.5), for $n=0,1,2,19,20,21$. This figure shows that successive applications of the map $\mathscr{F}$ on the discontinuous initial function $\psi_{0}(\tau)=x_{-}+\left(x_{+}-x_{-}\right) H(\tau)$, produces increasingly smooth iterates. For large $n$ the iterates are, modulo a phase shift, periodic with period two, that is $\psi_{n+2}(\tau)=\psi_{n}(\tau+2 s)$ for some shift, which we write as $2 s$. Note that in the right-hand panel of Figure 3 , for larger $\lambda$, the transition layers are not monotone, just before the descending layer starts its descent, it first increases and slightly exceeds the level of the plateau. Thus, in this parameter regime, the fast map (2.5) exhibits periodic behaviour with a period of two with a shift, $s$; that is

$$
\begin{aligned}
\psi_{2 n}(\tau) & \rightarrow \widetilde{\psi}(\tau-2 n s) \quad \text { as } n \rightarrow \infty, \\
\psi_{2 n+1}(\tau) & \rightarrow \widetilde{\phi}(\tau-(2 n+1) s) \quad \text { as } n \rightarrow \infty .
\end{aligned}
$$

and our aim now is to find the form of the functions $\widetilde{\psi}, \widetilde{\phi}$.

\subsection{Instability of the fixed point of the fast map}

The fast map (2.5) has a fixed point $\psi(\tau)=1-\lambda^{-1}$, which is the same as the fixed point of the onedimensional map (1.2). To investigate the stability of the fixed point of the fast map, we introduce

$$
\psi_{n}(\tau)=1-\lambda^{-1}+\delta \zeta_{n}(\tau)
$$

with $\delta \ll 1$ and linearising yields

$$
\zeta_{n+1}(\tau)=\mathscr{L}\left[\zeta_{n}(\tau)\right]:=-(\lambda-2) \mathrm{e}^{-\tau} \int_{s=-\infty}^{\tau} \mathrm{e}^{s} \zeta_{n}(s) \mathrm{d} s
$$

Since this equation is a linear difference equation in $n$, it has solutions of the form $\zeta_{n}(\tau)=\rho^{n} \zeta(\tau)$, where $\mathscr{L}[\zeta(\tau)]=\rho \zeta(\tau)$. Due to the form of the operator $\mathscr{L}$, its eigenfunctions have the form $\zeta(\tau)=\mathrm{e}^{i \omega \tau}$. An 

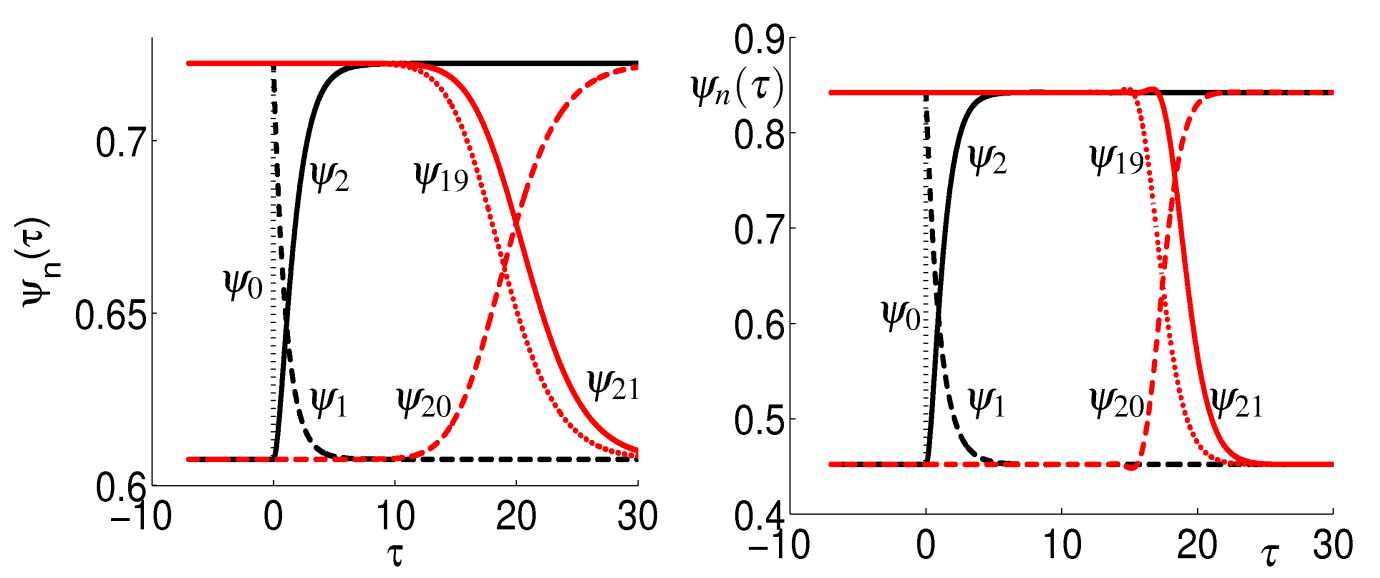

Figure 3: Illustration of the iterates $\psi_{n}(\tau)$ plotted against $\tau$ for $n=0,1,2,19,20,21$ for the case of $\lambda=$ 3.03 (left panel) and $\lambda=3.4$ (right panel). In both panels, $\psi_{0}$ is the left-most dotted line, $\psi_{1}$ is the left dashed line, $\psi_{2}$ is the left-most solid line, $\psi_{19}$ is the right dotted line, $\psi_{20}$ is the right dashed line, $\psi_{21}$ is the right solid line. The curves corresponding to $n=0,1,2$ have transitions centred on $0<\tau<5$ (black curves, on the left side of the graph); whilst those with transitions around $\tau \approx 20$ correspond to $n=19,20,21$, (red curves on the right). The online version is in colour.

instability occurs when $|\rho|>1$. The eigenvalues are given by $\rho=-(\lambda-2)(1-i \omega) /\left(1+\omega^{2}\right)$, which have magnitude $|\rho|=(\lambda-2) / \sqrt{1+\omega^{2}}$. Maximising $|\rho|$ over $\omega$ to find the first unstable mode, we obtain $\omega=0$, corresponding to $\zeta(\tau)=1$ and $\rho=-(\lambda-2)$. Thus, as $\lambda$ increases through the value $\lambda_{c}=3, \rho$ decreases through -1 and there is a period-doubling bifurcation.

After the bifurcation in the nonlinear equation (2.1), the derivative term is small and, to ensure it is involved in the leading order balance, we require $\partial_{\tau}=\mathscr{O}(\delta)$, hence we introduce a long timescale given by $T=\delta \tau$.

\subsection{Two-cycle of the fast map}

The period-two oscillation of the solution of the DDE corresponds to a two-cycle of the fast map, by which we mean that the second iterate of the fast map corresponds to a shift in the wave form, with no change in shape. That is, $\psi_{n+2}(\tau-2 s)=\psi_{n}(\tau)$ for some shift $s$, so that if, say, $\psi_{n}(0)=1-1 / \lambda$ then $\psi_{n+2}(2 s)=1-1 / \lambda$, that is, the crossing of the unstable fixed point $x=1-1 / \lambda$ will occur at larger $\tau$ when $n$ is larger; equivalently $\psi_{n+2}(\tau)=\psi_{n}(\tau+2 s)$ or $\mathscr{F}[\mathscr{F}[\psi(\tau)]]=\psi(\tau+2 s)$.

From the numerics shown in Figure 3, we observe that

$$
\psi_{2 n}(\tau+2 n s) \rightarrow \psi(\tau), \quad \psi_{2 n+1}(\tau+2 n s+s) \rightarrow \phi(\tau), \quad \text { as } n \rightarrow \infty, \text { for fixed } \tau .
$$

Estimating $s=1$, we obtain the results plotted in Figure 4. We observe that this leads to an almost complete cancellation of the shift: $\psi_{21}(\tau+21)$ is close to being coincident with $\psi_{19}(\tau+19)$; however, there is still some difference, hence $s$ is not exactly unity. This estimate of $s=1$ in fact slightly overcompensates for the shift. In the following section, we use asymptotic techniques to extract a more accurate expression for the shift, $s$, and obtain explicit approximations for the shape of the transition regions, $\psi(\tau)$ and $\phi(\tau)=\mathscr{F}[\psi(\tau-s)]$. In particular, note that figure 4 shows that the transition layers are nonmonotone at larger values of $\lambda$. Both the increasing and the decreasing layers explore the region outside that bounded by the two plateaus, see in particular the region around $\tau \approx-6$. 

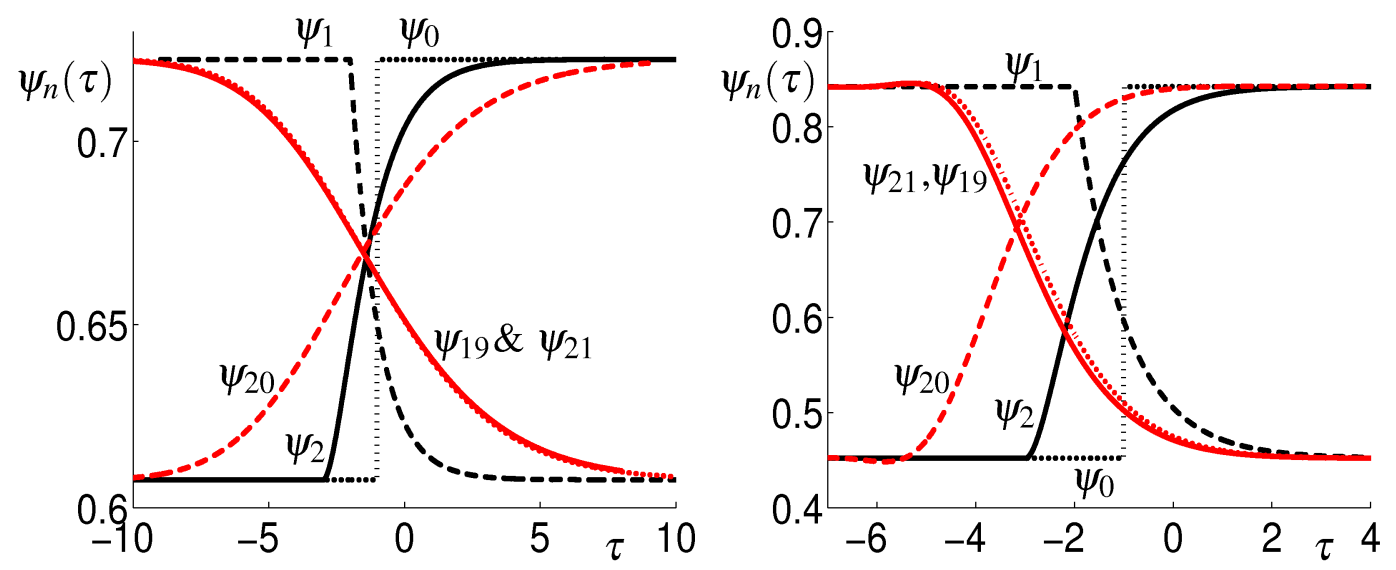

Figure 4: Illustration of the iterates $\psi_{n}(\tau-n)$ plotted against $\tau$ for $n=0,1,2,19,20,21$ for the case of $\lambda=3.03$ (left) and $\lambda=3.4$ (right). In both panels, the dotted square wave represents $\psi_{0}$, whilst $\psi_{1}$ is the steeper dashed curve containing a corner, and $\psi_{2}$ is the steeper solid curve; $\psi_{19}$ is the other dotted line, $\psi_{20}$, the other dashed curve, and $\psi_{21}$, the other solid curve, almost coincident with $\psi_{19}$. Note the different scales on the horizontal axes. The transition layers for $\lambda=3.03$ are considerably more slowly varying than those for $\lambda=3.4$; also note that at larger $\lambda$, there is greater asymmetry in the shape of the transition layers. Left panel: the more slowly-varying curves correspond to $n=19,20,21$, whilst the steeper curves illustrate $n=0,1,2$. Right panel: the layers corresponding to $n=0,1,2$ are centred at $-2<\tau<0$ are shown in red, whilst those for $n=19,20,21$ are centred on $\tau \approx-3$ and are shown in black. The online version is in colour.

\section{Asymptotic approximation of the 2-cycle of the fast map}

\subsection{Problem formulation}

Given the form of the two-cycle (1.3), we put

$$
\lambda=3+\delta^{2}
$$

and write the transition layers as

$$
\psi_{n}(\tau)=M+\alpha \Psi_{n}(\tau)
$$

where

$$
M=\frac{\lambda+1}{2 \lambda}=\frac{4+\delta^{2}}{6+2 \delta^{2}}, \quad \alpha=\frac{\sqrt{(\lambda+1)(\lambda-3)}}{2 \lambda}=\frac{\delta \sqrt{4+\delta^{2}}}{6+2 \delta^{2}}
$$

So that $\Psi_{n} \rightarrow \pm 1$ or $\mp 1$ as $\tau \rightarrow \pm \infty$. To be precise, we require $\Psi_{n}( \pm \infty)= \pm(-1)^{n}$ so that if $n$ is even then $\Psi_{n}$ is an 'up'-layer (that is, increasing), and if $n$ is odd, then $\Psi_{n}$ is a 'down'-layer, (decreasing).

Note that in this subsection, no assumption is made about the magnitude of $\delta$. Only in the next subsection (§3.2) do we assume that $\delta$ is small. The effect of the fast map, which determines one transition layer as a function of the previous layer is given by

$$
\frac{\mathrm{d} \Psi_{n+1}}{\mathrm{~d} \tau}+\Psi_{n+1}+\Psi_{n}=\frac{1}{2} \delta\left(1-\Psi_{n}^{2}\right) \sqrt{4+\delta^{2}}
$$

To analyse the two-cycle, with some phase shift $s$, we introduce

$$
\Psi(\tau)=\lim _{n \rightarrow \infty} \Psi_{2 n}(\tau+2 n s), \quad \Phi(\tau)=\lim _{n \rightarrow \infty}-\Psi_{2 n+1}(\tau+2 n s+s),
$$

so that both transition layer functions $\Psi(\tau), \Phi(\tau)$ are increasing. The boundary conditions are thus $\Phi( \pm \infty)=\Psi( \pm \infty)= \pm 1$. 
Requiring $\Psi_{2 n+2}$ to equal $\Psi_{2 n}$ modulo the phase shift, $s$, means that the shape of the transition layers are governed by the coupled pair of differential-delay equations

$$
\begin{aligned}
\Psi(\tau-s)+\Psi^{\prime}(\tau-s)-\Phi(\tau) & =\frac{1}{2} \delta\left(1-\Phi(\tau)^{2}\right) \sqrt{4+\delta^{2}} \\
\Phi(\tau-s)+\Phi^{\prime}(\tau-s)-\Psi(\tau) & =-\frac{1}{2} \delta\left(1-\Psi(\tau)^{2}\right) \sqrt{4+\delta^{2}}
\end{aligned}
$$

where the determination of $s$ is part of the problem. Next we write

$$
\Psi(\tau)=\xi(\tau)+\zeta(\tau), \quad \text { and } \quad \Phi(\tau)=\xi(\tau)-\zeta(\tau),
$$

so that $\xi(\tau)=\frac{1}{2}(\Phi(\tau)+\Psi(\tau))$ is the average shape of the transition layer, and $\zeta(\tau)=\frac{1}{2}(\Psi(\tau)-\Phi(\tau))$ accounts for the asymmetry in the shape of the layers. The quantities $\xi(\tau), \zeta(\tau)$ are governed by

$$
\begin{aligned}
& \xi(\tau-s)+\xi^{\prime}(\tau-s)-\xi(\tau)=\delta \xi(\tau) \zeta(\tau) \sqrt{4+\delta^{2}} \\
& \zeta(\tau-s)+\zeta^{\prime}(\tau-s)+\zeta(\tau)=\frac{1}{2} \delta\left(1-\xi(\tau)^{2}-\zeta(\tau)^{2}\right) \sqrt{4+\delta^{2}}
\end{aligned}
$$

together with the boundary conditions $\xi( \pm \infty)= \pm 1$ and $\zeta( \pm \infty)=0$.

Thus far, we have not made any use of asymptotic approximations, beyond the fast map in Section 2.2, nor have we assumed $\Phi(\tau)=\Psi(\tau)$, or $\Phi(\tau), \Psi(\tau), \xi(\tau)$ have odd symmetry, or $\zeta(\tau)$ is even.

\subsection{Asymptotic expansion}

We now make use of the approximation $\delta \ll 1$. Equation (3.10) implies $\zeta=\mathscr{O}(\delta)$, however, for the simplicity of later calculations we introduce a slightly modified small parameter, $v$, and write

$$
v=\frac{1}{2} \delta \sqrt{4+\delta^{2}}, \quad T=v \tau, \quad \xi(\tau)=\theta(T), \quad \zeta(\tau)=v \eta(T), \quad \text { with } \theta, \eta=\mathscr{O}(1) .
$$

We note that the boundary conditions $\xi( \pm \infty)= \pm 1$ and $\zeta( \pm \infty)=0$ imply

$$
\begin{aligned}
& \theta(T) \rightarrow 1 \quad \text { as } T \rightarrow \infty, \quad \theta(T) \rightarrow-1 \quad \text { as } T \rightarrow-\infty \\
& \eta(T) \rightarrow 0 \text { as } T \rightarrow \pm \infty .
\end{aligned}
$$

Thus equations (3.9)-(3.10) imply

$$
\begin{aligned}
& \theta(T-v s)+v \theta^{\prime}(T-v s)-\theta(T)=2 v^{2} \theta(T) \eta(T) \\
& \eta(T-v s)+v \eta(T-v s)+\eta(T)=1-\theta(T)^{2}-v^{2} \eta(T)^{2} .
\end{aligned}
$$

Note that if we just consider the leading order terms in (3.14), we obtain $\theta(T)-\theta(T)=0$. If we go to the next order terms, we find $(1-s) \theta^{\prime}(T)=0$, and since $\theta^{\prime}=0$ is not a possible solution, we require $s=1$, to leading order. However, this has still not generated an approximation for $\theta(T)$. To proceed further, we expand the delay $s$ as

$$
s=S_{0}+v S_{1}+v^{2} S_{2}+\mathscr{O}\left(v^{3}\right)
$$

where $S_{0}=1$ has already been determined. We also write

$$
\theta(T)=\theta_{0}(T)+v \theta_{1}(T)+\ldots, \quad \text { and } \quad \eta(T)=\eta_{0}(T)+v \eta_{1}(T)+\ldots,
$$

where $\theta_{0}(T), \eta_{0}(T)$ are the leading order solutions. 


\subsection{Leading order approximation of the 2-cycle}

From the leading order terms in equation (3.15) it is clear that $\eta_{0}(T)=\frac{1}{2}\left(1-\theta_{0}(T)^{2}\right)$ and expanding equation (3.14) to $\mathscr{O}\left(v^{2}\right)$ yields

$$
v(1-s) \theta_{0}^{\prime}(T)+\frac{1}{2} v^{2} s(s-2) \theta_{0}^{\prime \prime}(T)=v^{2} \theta_{0}(T)\left(1-\theta_{0}(T)^{2}\right) .
$$

Using (3.16) with $S_{0}=1$ we obtain an autonomous problem for $\theta_{0}$, namely

$$
\theta_{0}^{\prime \prime}(T)+2 S_{1} \theta_{0}^{\prime}(T)=-2 \theta_{0}(T)\left(1-\theta_{0}(T)^{2}\right)
$$

Multiplying through by $\theta_{0}^{\prime}(T)$ and integrating from $T=-\infty$ to $T=+\infty$ we find $S_{1} \int_{-\infty}^{\infty} \theta_{0}^{\prime}(T)^{2} \mathrm{~d} T=0$; hence $S_{1}=0$ (since the integral must be strictly positive). This is the secularity condition required by the Fredholm alternative. The ODE (3.19) then simplifies and is solved by $\theta_{0}=\tanh (T)$. More generally, the dynamics of this equation can be understood using phase planes; the system has a centre at $\left(\theta_{0}, \theta_{0}^{\prime}\right)=$ $(0,0)$ and saddles at $\left(\theta_{0}, \theta_{0}^{\prime}\right)=( \pm 1,0)$. The homoclinic trajectory joining the two saddles is given by $\theta_{0}^{\prime}= \pm\left(1-\theta_{0}^{2}\right)$ and corresponds to the transition layers, which are our main interest here.

Retracing our steps to find leading order approximations for $\Phi(T)$ and $\Psi(T)$ we recover $\Psi(T), \Phi(T)=$ $\theta_{0}(T) \pm v \eta_{0}(T)$ and, since $\eta_{0}(T)=\frac{1}{2} \theta_{0}^{\prime}(T)$, the approximations for $\Psi(T)$ and $\Phi(T)$ are simply phase shifts of $\theta_{0}(T)$. Furthermore, since $s=1+\mathscr{O}\left(v^{2}\right)$, we have also shown that the phase shift is unity to leading order. In order to determine a more accurate approximation for the phase shift, we go to higher order in $v$, where we will also find a correction term for the transition layer. This more accurate shape will explain the overshooting behaviour and asymmetry of the shape of the layers seen in Figures 3 and 4.

\subsection{Higher-order terms}

Substituting the expansions (3.17) into (3.14)-(3.15), and recalling that the delay term (3.16) simplifies to $s=1+v^{2} S_{2}$, we obtain the equations

$$
\begin{gathered}
\theta_{0}^{\prime \prime}(T)=-4 \theta_{0}(T) \eta_{0}(T), \quad 2 \eta_{0}(T)=1-\theta_{0}(T)^{2}, \quad \eta_{1}(T)=-\theta_{0}(T) \theta_{1}(T), \\
\frac{1}{2} \theta_{1}^{\prime \prime}(T)+2 \eta_{0}(T) \theta_{1}(T)=\frac{1}{3} \theta_{0}^{\prime \prime \prime}(T)-2 \theta_{0}(T) \eta_{1}(T)-S_{2} \theta_{0}^{\prime}(T) .
\end{gathered}
$$

Using the leading order solutions $\theta_{0}=\tanh (T), \eta_{0}=\frac{1}{2} \operatorname{sech}^{2}(T)$ to simplify (3.21), we obtain

$$
\theta_{1}^{\prime \prime}+2 \theta_{1}\left(1-3 \tanh ^{2} T\right)=2\left(1-\tanh ^{2} T\right)\left(2 \tanh ^{2} T-\frac{2}{3}-S_{2}\right) .
$$

As before, to find the correction to the phase shift, $S_{2}$, we multiply through by $\theta_{0}^{\prime}$ and integrate over $T$, to obtain

$$
S_{2}=-\int_{-\infty}^{\infty} 2 \operatorname{sech}^{4}(T)\left(2 \tanh ^{2}(T)-\frac{2}{3}\right) \mathrm{d} T / \int_{-\infty}^{\infty} 2 \operatorname{sech}^{4}(T) \mathrm{d} T=-\frac{4}{15} .
$$

Thus, for $0<\lambda-3 \ll 1$ the shift per iterate of the fast map can be approximated by

$$
s \sim 1-\frac{4}{15} v^{2} \sim 1-\frac{4}{15} \delta^{2} \sim 1-\frac{4}{15}(\lambda-3) .
$$

This explains why the unit shift applied between Figures 3 and 4 very slightly overcompensates for the shift.

Furthermore, the perturbation to the shape of the solution, $\theta_{1}(T)$, can be calculated explicitly. The complementary function for (3.22) has the form

$$
\theta_{1}(T)=A_{0} \operatorname{sech}^{2}(T)+B_{0} u(T), \quad u(T)=3 T \operatorname{sech}^{2}(T)+3 \tanh (T)+2 \sinh (T) \cosh (T),
$$



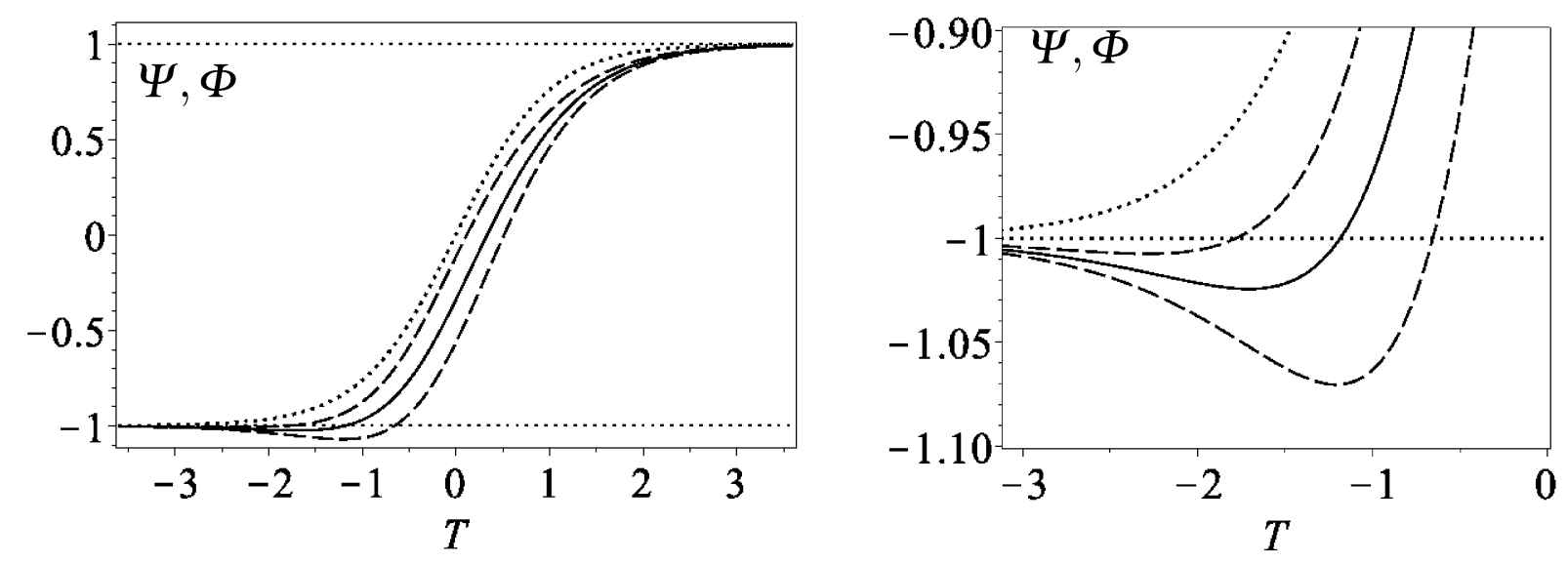

Figure 5: Illustration of the transition region functions $\Psi, \Phi$ plotted against $T$ including the first corrections to the tanh profile. The dotted lines represent $\Phi=\Psi= \pm 1$ and $\Phi=\Psi=\tanh (T)$; the solid line shows $\tanh (T)+v \theta_{1}(T)$, which is nonmonotone, having values below -1 for $T$ in the range for $T \lesssim-1.18$. Here we have chosen a reasonably large value of $v$, namely 0.46 to illustrate the behaviour. We also plot in dashed lines the functions $\tanh (T)+v\left[\theta_{1}(T) \pm 0.5 \operatorname{sech}^{2}(T)\right]$, which include the effect of $\eta_{0}=\frac{1}{2} \operatorname{sech}^{2}(T)$ in (3.29) and (3.30). At smaller values of $v$, this simply causes a phase shift in the profile, but this larger value of $v$ causes a more significant alteration to the shape. The right panel is a blow-up of the left, to show more clearly the effect of including the nonmonotonic, or 'overshooting' behaviour.

with $A_{0}, B_{0}$ being arbitrary constants. A particular solution can be constructed by writing

$$
\theta_{1}(T)=A(T) \operatorname{sech}^{2}(T)+B(T) u(T),
$$

and using the method of variation of parameters, which yields

$$
\begin{aligned}
& A(T)=A_{0}+\frac{4}{5} \log (\operatorname{sech}(T))+\frac{1}{10} \operatorname{sech}^{2}(T)\left[1-\frac{1}{3} \operatorname{sech}^{2}(T)+3 T \tanh (T) \operatorname{sech}^{2}(T)\right] \\
& B(T)=B_{0}-\frac{1}{10} \tanh (T) \operatorname{sech}^{4}(T) .
\end{aligned}
$$

Since the function $u(T) \sim \mathrm{e}^{2 T}$ as $T \rightarrow \infty$, and we require boundary conditions in which $\theta_{1} \rightarrow 0$ as $T \rightarrow \pm \infty$, we choose $B_{0}=0$. The combination $B(T) u(T)$ then decays to zero as $T \rightarrow \infty$, with $B(T) u(T) \sim \mathscr{O}\left(\mathrm{e}^{-2 T}\right)$. The constant $A_{0}$ is left arbitrary, as adding a small component, namely $v A_{0} \operatorname{sech}^{2}(T)$, to the leading order $\theta_{0}(T)=\tanh (T)$ solution merely corresponds to a phase shift, namely $\theta_{0}\left(T+v A_{0}\right)=\tanh \left(T+v A_{0}\right)$. Note that while $A(T)$ grows linearly with $T$ as $T \rightarrow \infty$, the combination $A(T) \operatorname{sech}^{2}(T)$ is bounded. This product has the asymptotic decay of $\theta_{1} \sim T \mathrm{e}^{-2 T}$ as $T \rightarrow \infty$. The decay of this perturbation is thus slightly slower than that of the leading order term, whose asymptotic behaviour is $\tanh (T) \sim 1-\mathscr{O}\left(\mathrm{e}^{-2 T}\right)$.

Inverting the transformations (3.8) to regain $\Psi, \Phi$, we find

$$
\begin{aligned}
& \Psi(\tau)=\tanh (\nu \tau)+\frac{1}{2} \nu\left[2 \theta_{1}(\nu \tau)+\operatorname{sech}^{2}(v \tau)\right] \\
& \Phi(\tau)=\tanh (\nu \tau)+\frac{1}{2} v\left[2 \theta_{1}(\nu \tau)-\operatorname{sech}^{2}(\nu \tau)\right]
\end{aligned}
$$

These functions are plotted in Figure 5. Since, for small $\varepsilon$, the two-cycle exists for $3 \leq \lambda \leq 1+\sqrt{6}$, the maximum relevant value for $\delta$ is $\delta=0.449$, which yields a maximum value for $v$ of $v=0.46$, which is the value used in plotting Figure 5.

Relating our final time variable $T$ back to the original variable $t$, we find

$$
t-n=\varepsilon \tau=\frac{\varepsilon T}{v}=\frac{\varepsilon T}{\delta \sqrt{1+\delta^{2} / 4}} .
$$



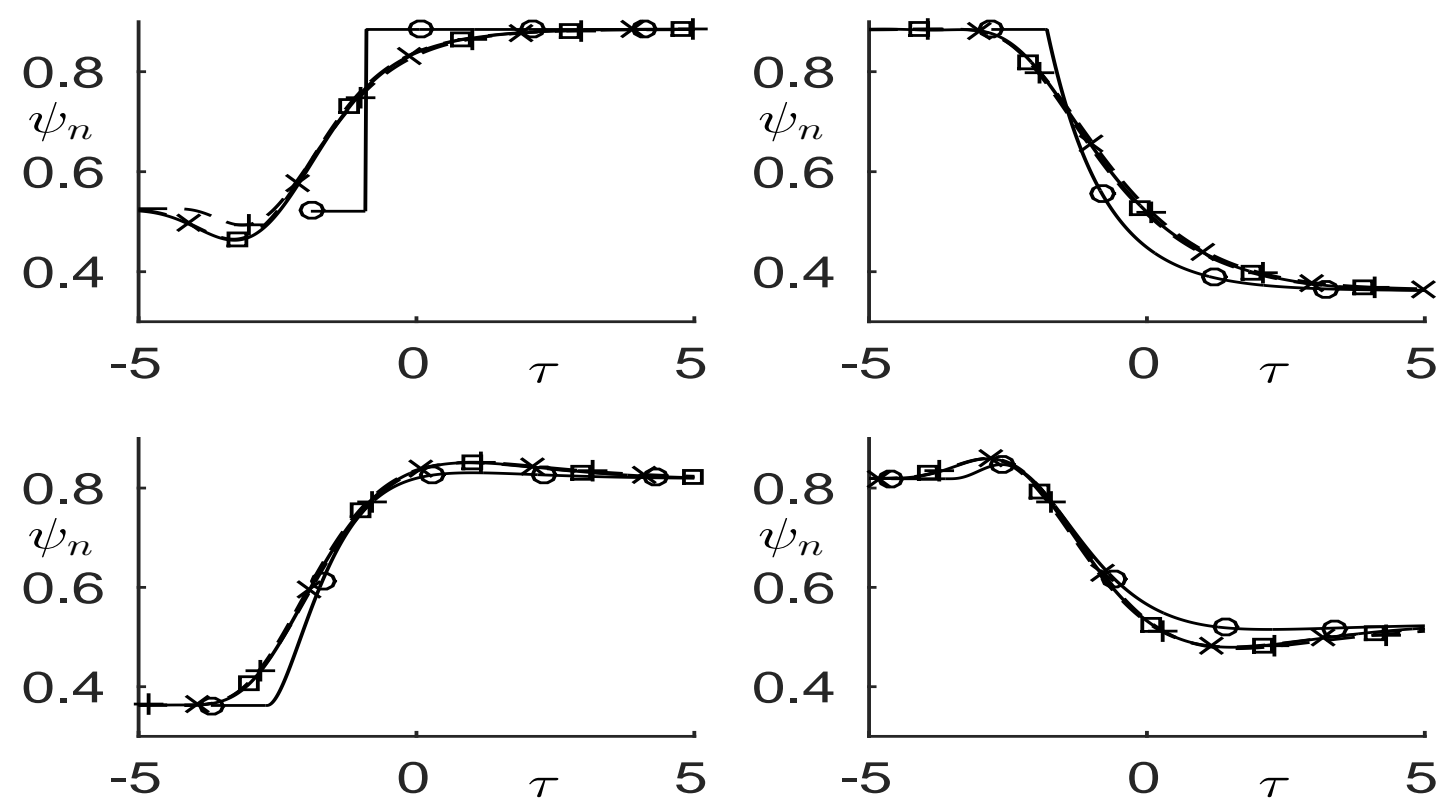

Figure 6: Illustration of the numerical solution of the fast map (2.1) when $\lambda=3.5$, corresponding to the 4-cycle of the logistic map. Top left, iterates 1, 5, 9, 13; top right, iterates 2, 6, 10, 14; bottom left, iterates $3,7,11,15$; bottom right, iterates $4,8,12,16$; in each panel the iterates are denoted in the order 'o', ' + ', ' $X$ ' and ' $\square$ '; in each panel the third and fourth iterates appear identical.

We are interested in points in the transition layer, where $T=\mathscr{O}(1)$ and $t-n$ is small. Since both $\delta$ and $\varepsilon$ are small parameters, and we expect the above expression to be small, we require $\varepsilon \ll \delta \sqrt{1+\delta^{2} / 4} \ll 1$. In the next section we explore cycles with longer periods numerically.

\section{Transition layers in the period 3 and 4 cycles}

In Figure 6 we plot the transition layers for the four cycle as determined by a numerical solution of (2.1). In the logistic map the four-cycle is stable for $1+\sqrt{6} \approx 3.449<\lambda<3.544$. Results are presented for the case $\lambda=3.5$ which is in the centre of this parameter range; the plateaus are given by $x_{1}=0.521$, $x_{2}=0.884, x_{3}=0.362, x_{4}=0.819$. We apply a numerically determined horizontal shift of $s=0.9$ to the results to show the convergence in shape of transition layers at later iterates. Although six curves are plotted in each panel, most of those corresponding to later iterates cannot be seen as they lie on top of each other. The top left panel shows undershooting of the layer $\left(\psi<x_{1}\right)$ before converging to the higher plateau, $\psi=x_{2}$, whilst the layer plotted in the lower right panel exhibits both overshooting and undershooting, that is, $\psi>x_{4}$ and $\psi<x_{1}$ for differing $\tau$. Thus much of the behaviour discussed in Sections 2.3, 2.5 and 3 persists in a qualitative fashion for the period four cycle.

Figure 7 shows the corresponding results for the three-cycle. Here there is no convergence in shape of the transition layers. Instead, with each iterate, the layers increase in width, gaining additional internal oscillations. If we attempt to find a shift to superimpose layers on top of each other, there are two natural choices. Firstly, using $s=0.35$, the start of each layer can be made to coincide, as shown in the left panels of Figure 7. The shape of the start of the transition layers show no similarity once the move away from the initial plateau has occurred. More interestingly, a value of $s=2$ leads to the right-hand panels of Figure 7, where the right-hand edges of each layer are superimposed. This shows greater similarity in the shape of the later parts of the transition layer. For example, note that the dashed and solid black lines are coincident not just in the final convergence to the plateaus, but also for several oscillations beforehand, as shown by iterates 19 and 22 in the top right panel, iterates 20 and 23 in the middle right panel, and iterates 21 and 24 in the lower right panel. 

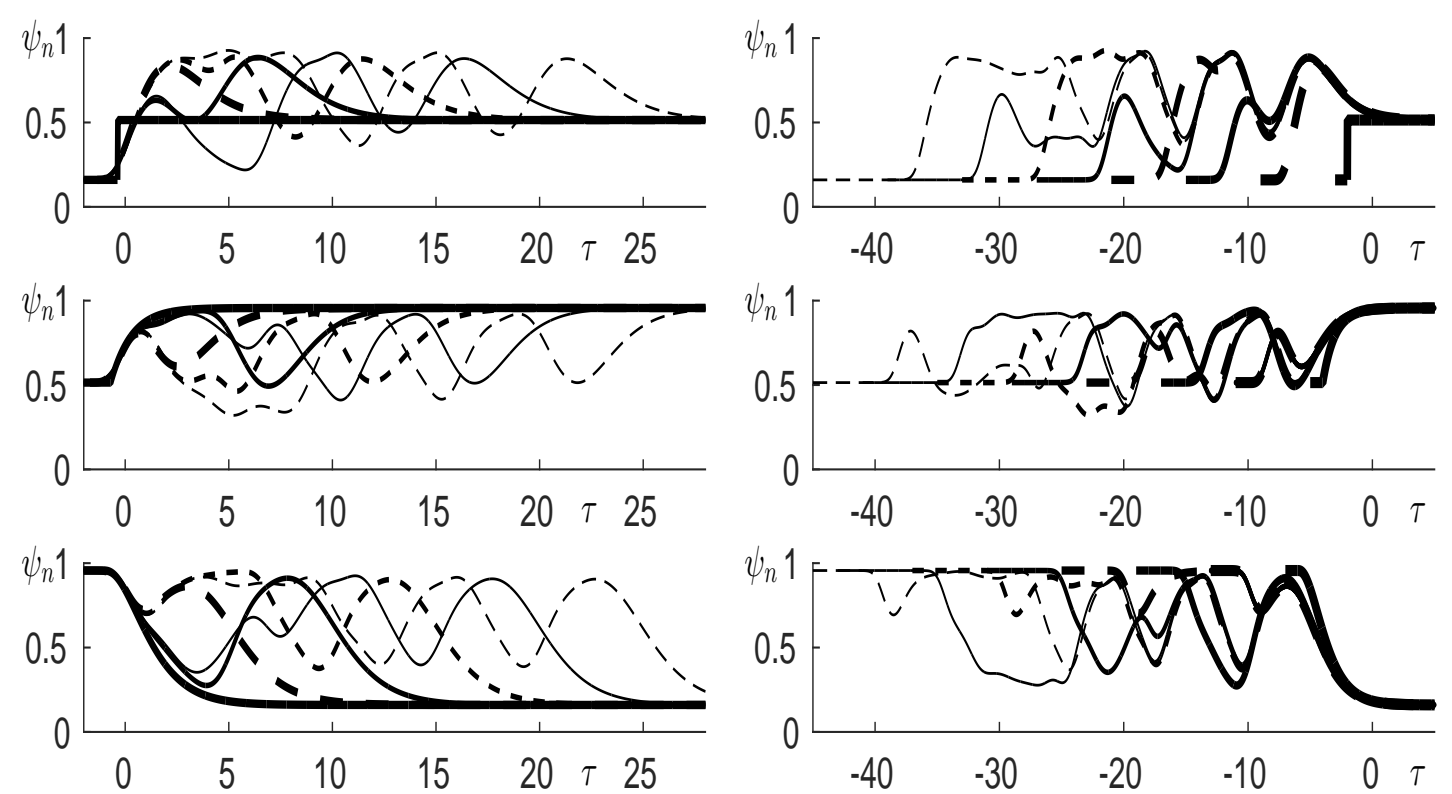

Figure 7: Illustration of the numerical solution for the fast map (2.1) when $\lambda=1+2 \sqrt{2}$, corresponding to the 3-cycle of the logistic map. On the left, a shift of $s=0.35$ is applied so that the left hand edges of the transition layers coincide. Top left, iterates 1, 4, 7, 10, 13, 16; middle left: iterates 2, 5, 8, 11, 14, 17; bottom left: iterates $3,6,9,12,15,18$; in each case, the order in which the iterates are plotted is given by solid thick line, dashed thick line, medium solid line, medium dashed line, thin solid line and thin dashed lines. In the right-hand panels, a shift of $s=2$ is applied so that the right edge of the transition layers coincide. Top right: iterates $1,4,7,10,13,16,19,22$; middle right: iterates 2, 5, 8, 11, 14, 17, 20, 23; lower right: iterates $3,6,9,12,15,18,21,24$; in each case the order in which the iterates are plotted is given by: very thick solid line, very thick dashed line, thick solid line, thick dashed line, medium solid line, medium dashed line, narrow solid line, narrow dashed line.

\section{Conclusions}

Any differential-delay equation of the form (1.1) which undergoes a bifurcation whereby the steady-state becomes unstable can be approximated by a quadratic. The analysis of the logistic map thus has a wider relevance to singularly perturbed differential-delay equations. We have studied such a differential-delay equation which, in the singular limit of small $\varepsilon$, reduces to the well-known one-dimensional logistic map. We have shown that as the 1D map undergoes a bifurcation to a period two state, so does the delay equation.

The analysis of Section 1.2 results in the formula (1.10) for the period of the oscillation on the bifurcation curve. This formula only predicts the period of oscillation on the bifurcation curve (1.7), and is only valid for the case where harmonic solutions are produced, that is, away from the limit $0<\varepsilon \ll 1$.

The solution of the singularly perturbed delay equation (1.1) has plateaus of approximately unit length, separated by narrow transition layers. In Section 3 we study the bifurcation which occurs as $\lambda$ is increased through the value $\lambda=3$ with $\varepsilon \ll 1$. In this case, square wave solutions are produced, and the period depends on both $\lambda$ and $\varepsilon$, and these parameters are treated independently in the result (5.1) which holds for more general $\lambda-3 \ll 1, \varepsilon \ll 1$.

By introducing the 'fast map', which is an approximate Poincaré map, relating the form of each transition layer to the previous one, we have generated a further system of differential delay equations for the shape of the transition layers in the period-two cycle (3.7). In this system, the delay is an unknown parameter, for which we have generated an asymptotic expansion. The first few terms of this expansion are given in (3.24). In the original time variable $(t)$ the period is

$$
P=2+2 \varepsilon s=2+2 \varepsilon-\frac{8}{15} \varepsilon(\lambda-3) .
$$


This agrees with the result derived by Adhikari et al. (2008). For any particular choice of parameters, the period of the periodic solution depends on both $\lambda$ and $\varepsilon$.

Analysing the fast map using asymptotic techniques, we have shown that, to leading order, the layers have a tanh shape, as might be expected. More significantly, in section 3.4 we have shown that the higher order perturbation terms give rise to more complex behaviour. In particular, the profile has an asymmetric shape, with nonmonotonic behaviour, and slower convergence to the plateaus than the leading order solution suggests. All these effects become more pronounced as $\lambda$ increases beyond the bifurcation point $\lambda=3$.

The form of the transition layers have been further explored through a numerical solution of the fast map in the cases of the four cycle and three cycle of the logistic map. In the four cycle, the transition layers again rapidly converge to one of the four steady shapes. Denoting the plateaus by $x_{1}, x_{2}, x_{3}, x_{4}$, there are four attracting shapes for the transition layers, one for each of the $x_{1}-x_{2}, x_{2}-x_{3}, x_{3}-x_{4}$, and $x_{4}-x_{1}$ layers illustrated in Figure 6. The transition layers between the three-cycle plateaus, however, do not converge to a steady form. Instead, they grow in width, whilst showing some convergence in the shape of their right-hand edges. This increasing complexity provides considerable challenges for more theoretical analyses.

\section{Acknowledgements}

I am grateful to Andrew Fowler and Guy Kember for their advice during the preparation of my master's thesis, Wattis (1990), and to the UK SERC for funding my studies. More recently, my thanks go to Louise Adams for performing preliminary numerical simulations. Helpful suggestions from referees have also improved the manuscript, including highlighting the papers by Nussbaum (1982), Mallet-Paret \& Nussbaum (1993), and Adhikari et al. (2008) which contain some analysis similar to that presented here.

\section{References}

Adhikari, M.H., Coutsias, E.A., McIver, J.K. (2008) Periodic solutions of a singularly perturbed delay differential equation. Physica D 237, 3307-3321.

ArfKen, G. (1985) Mathematical Methods for Physicists. Academic press, Orlando, Fl, USA

Chow, S.N., HAle, J.K., \& HuAnG, W. (1992) From sine waves to square-waves in delay equations, Proc. Roy. Soc. Edinburgh A 120, 223-229.

Chow, S.N., Lin, X.B., \& Mallet-Paret, J. (1989) Transition layers for singularly perturbed delay differential equations with monotone nonlinearities. J Dynamics and Differential Equations, 1, 3-43.

Chow. S.N. \& MAllet-Paret, J. (1983) Singularly perturbed delay-differential equations, in 'Coupled Nonlinear Oscillators.' (ed. Chandra. J. \& Scott, A.C.) North Holland Math. Studies 80 pp. 7-12.

Erneux, T., Larger, L., Lee M.W., Goedgebuer, J-P (2004) Ikeda Hopf bifurcation revisited. Physica D 194, 49-64.

Fowler, A.C. (1997) Mathematical Models in the Applied Sciences. Cambridge, CUP.

FOWLER, A.C. (1982) An asymptotic analysis of the delayed logistic equation when the delay is large. IMA J Appl Math. 28, 41-49.

Fowler, A.C., \& MACKey, M.C. (2002) Relaxation oscillations in a class of delay differential equations. SIAM J Appl Math 63, 299-323. 
GibBS, J.W. (1898) Fourier's series. Nature, 59, 200.

GiBBS, J.W. (1899) Fourier's series. Nature, 59, 606.

Glass, L. \& Mackey, M.C. (1988) From Clocks to Chaos. Princeton University Press, (pp. 68-78).

Gurney, W.S., Blythe, S.P., \& Nisbet, R.M. (1980) Nicholson's blowflies revisited. Nature, 287, $17-21$.

HALE, J.K. \& HuAng, W. (1994) Period-doubling in singularly perturbed delay equations. J. Diff. Equ. 114, 1-23.

Holton, D. \& May, R.M. (1993) Chaps 5-8 of Mullin, T.,The Nature of Chaos, Oxford, OUP.

IKEDA, I. (1979) Multiple-valued stationary state and its instability of the transmitted light by a ring cavity system. Opt. Commun. 30, 257-261.

IKeDA, I. (1985) Delay-Differential Equations Modelling Nonlinear Optical Resonators, in 'Optical Instabilities' (ed. Boyd, R.W. et al.) pp. 85-98.

IKEDA, I., DAIDO, H., \& AKImoto, O. (1980) Optical turbulence: chaotic behaviour of transmitted light from a ring cavity. Phys. Rev. Lett., 45, 709-712.

Mackey, M.C. (1979) Periodic auto-immune hemolytic anemia: an induced dynamical disease. Bull. Math. Biol., 41, 829-834.

MACKEY, M.C. \& Glass, L. (1977) Oscillations and chaos in physiological control systems. Science 197, 287-289.

Mallet-Paret, J. \& Nussbaum, R.D. (1986) Global continuation and complicated trajectories for periodic solutions of a differential-delay equation. Proc. Symp. Pure Math., Am. Math. Soc. 45 (pt.2), 155-167.

Mallet-Paret, J. \& Nussbaum, R.D. (1986) Global continuation and asymptotic behaviour for periodic solutions of a differential-delay equation. Ann. Mat. Pura ed Appli. 145, 33-128.

Mallet-Paret, J. \& Nussbaum, R.D. (1989) A differential-delay equation arising in optics and physiology. SIAM J. Math. Anal. 20, 249-292.

Mallet-Paret, J. \& Nussbaum, R.D. (1993) Multiple transition layers in a singularly perturbed differential-delay equation. Proc. Roy. Soc. Ed. 123A, 1119-1134.

MAY, R.M. (1976) Simple mathematical models with very complicated dynamics Nature 261, 459-467.

Nizette, M. (2003) Front dynamics in a delayed-feedback system with external forcing. Physica D $183,220-244$.

Nussbaum, R.D. (1982). Asymptotic analysis of some functional differential equations. Pp. 277-302 of Bednarik, E and Cesari, L., Dynamical Systems II, an International Symposium, Academic Press, New York.

Wazewska-Czysewska, M. \& Lasota, A. (1976) Mathematical Models of the Red Cell System. Matemnatyka Stosowana, 6, 23-40.

WATTIS, J.A.D. (1990) Bifurcations and Chaos in a Differential-Delay Equation. MSc thesis, Univ of Oxford, UK. 